\title{
Intentional Laceration of the Anterior Mitral Valve Leaflet to Prevent Left Ventricular Outflow Tract Obstruction During Transcatheter Mitral Valve Replacement:
}

\section{Pre-Clinical Findings}

Jaffar M. Khan, BM, BCh ${ }^{\mathrm{a}}$, Toby Rogers, BM, BCh ${ }^{\mathrm{a}}$, William H. Schenke, BS ${ }^{\mathrm{a}}$, Jonathan R. Mazal, MS ${ }^{a}$, Anthony Z. Faranesh, PhD $^{a}$, Adam B. Greenbaum, MD ${ }^{b}$, Vasilis C. Babaliaros, MD $^{\mathrm{C}}$, Marcus Y. Chen, MD ${ }^{\mathrm{a}}$, and Robert J. Lederman, MD ${ }^{\mathrm{a}}$

aCardiovascular and Pulmonary Branch, Division of Intramural Research, National Heart, Lung, and Blood Institute, National Institutes of Health, Bethesda, Maryland benter for Structural Heart Disease, Division of Cardiology, Henry Ford Health System, Detroit, Michigan 'Structural Heart and Valve Center, Emory University Hospital, Atlanta, Georgia

\section{Abstract}

OBJECTIVES-The authors propose a novel transcatheter transection of the anterior mitral leaflet to prevent iatrogenic left ventricular outflow tract (LVOT) obstruction during transcatheter mitral valve replacement (TMVR).

BACKGROUND—LVOT obstruction is a life-threatening complication of TMVR caused by septal displacement of the anterior mitral leaflet.

METHODS-In vivo procedures in swine were guided by biplane x-ray fluoroscopy and intracardiac echocardiography. Retrograde transaortic 6-F guiding catheters straddled the anterior mitral leaflet. A stiff 0.014-inch guidewire with polymer jacket insulation was electrified and advanced from the LVOT, through the A2 leaflet base, into the left atrium. The wire was snared and externalized, forming a loop that was energized and withdrawn to lacerate the anterior mitral leaflet.

RESULTS-The anterior mitral leaflet was successfully lacerated in 7 live and 1 post-mortem swine under heparinization. Lacerations extended to $89 \pm 19 \%$ of leaflet length and were located within $0.5 \pm 0.4 \mathrm{~mm}$ of leaflet centerline. The chordae were preserved and retracted the leaflet halves away from the LVOT. LVOT narrowing after benchtop TMVR was significantly reduced with intentional laceration of the anterior mitral leaflet to prevent LVOT obstruction than without ( $65 \pm 10 \%$ vs. $31 \pm 18 \%$ of pre-implantation diameter, $\mathrm{p}<0.01$ ). The technique caused mean

REPRINT REQUESTS AND CORRESPONDENCE: Dr. Robert J. Lederman, Cardiovascular and Pulmonary Branch, Division of Intramural Research, National Heart, Lung, and Blood Institute, National Institutes of Health, Building 10, Room 2c713, MSC 1538, Bethesda, Maryland 20892-1538. lederman@nih.gov.

All other authors have reported that they have no relationships relevant to the contents of this paper to disclose.

APPENDIX For a supplemental video, please see the online version of this article. 
blood pressure to fall (from $54 \pm 6 \mathrm{~mm} \mathrm{Hg}$ to $30 \pm 4 \mathrm{~mm} \mathrm{Hg}, \mathrm{p}<0.01$ ), but blood pressure remained steady until planned euthanasia. No collateral tissue injury was identified on necropsy.

CONCLUSIONS-Using simple catheter techniques, the anterior mitral valve leaflet was transected. Cautiously applied in patients, this strategy can prevent anterior mitral leaflet displacement and LVOT obstruction caused by TMVR.

\section{Keywords}

left ventricular outflow tract obstruction; mitral valve; structural heart disease; subvalvular aortic stenosis; transcatheter mitral valve replacement; valvular heart disease

Transcatheter stent valves (both purpose-built and off-label) are implanted to relieve mitral valve failure — whether native, bioprosthetic, or after annuloplasty—when the risk for mitral valve surgery is prohibitive $(1,2)$. These transcatheter mitral stent valves may cause acute left ventricular outflow tract (LVOT) obstruction by displacing the anterior mitral valve leaflet toward the septum. Formal criteria have not been established, but as in surgery $(3,4)$, contributors to LVOT obstruction include angulated mitral and aortic annular planes, long or redundant anterior mitral leaflets, small ventricles, bulging septa, and narrow leaflet-toseptum distance (5-7). Preparatory or bailout transcoronary alcohol septal ablation can debulk the septum $(8,9)$ but risks important myocardial and conduction system injury. Moreover, alcohol septal ablation is not feasible when septal thickness is normal and typically requires a delay of 4 to 6 weeks for remodeling before transcatheter mitral valve replacement (TMVR), in highly symptomatic patients. The anterior mitral leaflet can be resected during hybrid surgical TMVR but requires cardiopulmonary bypass (10). We propose a trans-catheter alternative.

We describe a simple catheter technique to prevent LVOT obstruction by transecting the anterior mitral valve leaflet, called laceration of the anterior mitral leaflet to prevent LVOT obstruction (LAMPOON). The procedure uses an electrified guidewire that traverses the leaflet base, between 2 retrograde aortic catheters, and which then is pulled outward toward the leaflet tip (Figures 1A and 1B). The split anterior mitral leaflet no longer obstructs the LVOT after stent valve implantation and is displaced around the implant by intact chordae tendineae (Figures $1 \mathrm{C}$ to $1 \mathrm{~F}$ ). We developed and tested the technique in vivo and ex vivo in swine.

\section{METHODS}

\section{LAMPOON TECHNIQUE}

The technique has 2 steps: leaflet traversal followed by leaflet laceration (Figure 2).

Traversal is intended to be performed before, and laceration after, positioning of the transcatheter mitral valve. This would allow rapid valve implantation during expected hemodynamic compromise from intended mitral leaflet laceration.

For leaflet traversal, dual retrograde 6-F guiding catheters (Vista Brite Tip, Cordis Corporation, Miami Lakes, Florida) were positioned using 0.035-inch guidewires, 1 into the left atrium, taking care to cross the main mitral orifice without chordal entanglement, and 
the other in the LVOT, abutting the aortomitral curtain. The LVOT catheter was positioned immediately below the hinge point of the aortomitral curtain, as confirmed by contrast angiography in a projection that corresponds to a 3-plane echocardiogram. Alignment along the center of the anterior leaflet, corresponding to the commissure between the left and noncoronary cusps of the aortic valve, was achieved using contrast angiography in a projection corresponding to a short-axis echocardiogram. Intracardiac echocardiography (AcuNav; Siemens Healthcare, Erlangen, Germany) confirmed this position. A closed-loop snare (10-mm Amplatz GooseNeck, Medtronic, Minneapolis, Minnesota) was positioned through the left atrial catheter behind the atrial base of the anterior mitral leaflet. Through the LVOT catheter, a stiff 0.014-inch guidewire (Astato XS 20, Asahi-Intecc, Nagoya, Japan) was extended through an electrically insulating 0.035-inch polymer jacket (Piggyback Wire Convertor $145 \mathrm{~cm}$, Vascular Solutions, Minneapolis, Minnesota) and directed toward the snare. The proximal guidewire was connected via forceps to a monopolar electrosurgery pencil and diathermy generator (Valleylab Force FX, Medtronic, Minneapolis, Minnesota) set at 30-W continuous duty cycle ("cutting" mode). After traversal, the Piggyback polymer jacket was withdrawn, and the free end of the guidewire was externalized through the retrograde left atrial catheter, positioned to protect against inadvertent tissue injury. The result was a transcatheter guidewire loop around the anterior mitral valve leaflet. No traction was applied until the laceration procedure was initiated, to avoid causing or exacerbating mitral valve regurgitation. Correct traversal was confirmed by angiography through the LVOT catheter and by echocardiography.

Laceration entails traction on both ends of the guidewire that has crossed the leaflet base, during electrification. The intended result is longitudinal transection of the anterior mitral valve leaflet, so that 2 remaining flaps are displaced medially and laterally by the transcatheter mitral valve implant, without causing LVOT obstruction. To facilitate radiofrequency ablation energy delivery on the inner (cutting) side of the guidewire, a 5- to 10-mm section of the middle shaft of the 0.014-inch guidewire insulation was denuded by noncircumferential scalpel abrasion (Figure 3A) and then deliberately kinked to confine the denuded section to the inner curvature. The original insulating Piggyback is positioned to mark and abut 1 margin of the denuded shaft, locked in place, and then the kinked denuded section is positioned at the intended laceration site. The guiding catheter tips are apposed within 2 to $5 \mathrm{~mm}$ to provide mechanical and electric protection during laceration. The guidewire-catheter relationships are locked using torque devices, and the guidewire is clamped to an electrosurgery pencil. To lacerate, both locked guiding catheters are retracted during brief 2-step electrification. Retraction force was measured using a force meter (ZP-11, Imada, Northbrook, Illinois). Afterward, the guiding catheters are further apposed, and 1 guidewire limb is pulled through to allow catheter removal.

\section{ANIMAL PROCEDURES}

Nonsurvival procedures on Yorkshire swine (mean weight, $51 \pm 7 \mathrm{~kg}$ ) were approved by the institutional animal care and use committee and conducted according to contemporary National Institutes of Health guidelines. Bilateral percutaneous femoral artery and vein introducer sheaths were placed during isoflurane anesthesia with mechanical ventilation, and animals received intravenous heparin $(150 \mathrm{IU} / \mathrm{kg})$ to achieve an activated clotting time $>350$ 
s. Biplane x-ray fluoroscopy (Artis Zee, Siemens Healthcare) and intracardiac echocardiography (AcuNav 8.5-F, Siemens) guided procedures. Euthanasia and necropsy were performed hours after the procedure.

To test the consequences of wrong crossing along the aortomitral curtain, an intentionally high traversal and laceration was performed. To test whether flowing blood would obscure thermal injury, the procedure was also performed in another animal under heparinization, immediately after euthanasia.

At the conclusion of these nonsurvival experiments, animals were euthanized. In all animals, the mitral and aortic structures were examined carefully for thermal or mechanical injury. The laceration positions and lengths were recorded.

TAVR was not performed in these naive animals, absent a suitable fixation mechanism, and instead was performed ex vivo. Native LVOT length (from aortic root to mitral annulus) and minimum LVOT anteroposterior diameter were measured in explanted hearts following in vivo LAMPOON. Transcatheter heart valves (23-mm SAPIEN 3, Edwards Lifesciences, Irvine, California) were implanted at the benchtop at a 70:30 ventricular position across the annulus, and LVOT geometry was measured with and without LAMPOON modification.

\section{IN VITRO HEATING}

We performed infrared photography (FLIR E40, FLIR, Portland, Oregon) to test focal midshaft heating during electrification of the insulation-stripped guidewire. A 2-catheter and guidewire crossing system was partially submerged in a saline bath including the electrosurgery indifferent electrode and held in place by a steel clamp to simulate potential electric coupling with a trans-catheter mitral valve (Figure 3B).

\section{IMAGING AND DATA ANALYSIS}

Post-procedure contrast-enhanced CT was performed on a 320-row volume scanner (Aquilion One Vision, Toshiba, Tokyo, Japan). Surface renderings were generated on an image processing workstation (Vitrea version 6.7, Toshiba).

Data are expressed as mean \pm SD. LVOT diameters were compared, before and after simulated TMVR with and without LAMPOON, using 1-way analysis of variance and a Student $t$ test with Dunnett's correction for multiple comparisons (Prism version 6, GraphPad Software, La Jolla, California).

\section{RESULTS \\ IN VIVO FINDINGS}

The LAMPOON procedure was performed in 7 live animals and in 1 post-mortem animal under heparinization. A representative procedure is depicted in Figure 2. The procedure was successful in all animals. The mean procedure time was $55 \pm 22 \mathrm{~min}$, including imaging but excluding anesthesia and vascular access. LAMPOON caused mean blood pressure to fall by $44 \%$ (from $54 \pm 6 \mathrm{~mm} \mathrm{Hg}$ to $30 \pm 4 \mathrm{~mm} \mathrm{Hg}, \mathrm{p}<0.01$ ), as expected, but blood pressure remained steady until planned euthanasia for approximately 4 hours. Retraction force was 
high $(50 \mathrm{~N}, 5.1 \mathrm{~kg})$ with an intact and electrified lacerating guidewire in vivo; retraction force was reduced (to $15 \mathrm{~N}, 1.5 \mathrm{~kg}$ ) using a denuded cutting surface surrounded by an insulated polymer jacket.

In 1 animal, the traversal was intentionally performed "low" to simulate avoiding a calcified basal leaflet and traversed 52\% of the length of the A2 leaflet. In another animal, the traversal was intentionally performed "high" or above the aortomitral curtain, across the transverse sinus, into the left atrium, to test a serious complication. This caused a small pericardial effusion without hemodynamic change, and the animal was excluded from further analysis. No other animal had pericardial effusion after $2 \pm 1 \mathrm{~h}$ of survival.

Intracardiac echocardiography demonstrated cavitation microbubbles, as expected, during both the traversal and laceration steps of the procedure. Intracardiac echocardiography also demonstrated not only laceration but also splaying of the A2 mitral leaflet and acute mitral valve regurgitation, as expected, after LAMPOON (Figure 4A). Concomitantly, in vivo computed tomography demonstrated splitting and splaying of the A2 mitral valve leaflets (Figure 4B, Online Video 1).

\section{POST MORTEM FINDINGS}

Post mortem examination of leaflets revealed jagged transections of the A2 anterior mitral leaflet in all animals (Figure 5F). All transections were located between major chordal insertions, as intended. The transection lengths were $19 \pm 3 \mathrm{~mm}$ in leaflets that were $21 \pm 4$ $\mathrm{mm}$ long ( $89 \pm 19 \%$ of leaflet length). The average intercommissural distance was $32 \pm 6$ $\mathrm{mm}$; the average position of the laceration was $0.5 \pm 0.4 \mathrm{~mm}$ from the center of the anterior mitral leaflet, as intended. Leaflets were $0.9 \pm 0.1 \mathrm{~mm}$ thick and showed no visible eschar.

There was no necropsy evidence of injury to the aortic root or aortic valve, nor disruption of the mitral subvalvular apparatus, on any animal whether LAMPOON was performed in vivo or post mortem in situ.

\section{IN VITRO AND EX VIVO FINDINGS}

Infrared photography demonstrated that heat during the application of radiofrequency ablation energy emanated from a single location, the denuded portion of the guidewire suspended between insulated guiding catheters (Figure 3B). A nearby stainless steel clamp, positioned to simulate a potentially conductive metallic trans-catheter heart valve, did not exhibit heating, which would reflect radiofrequency coupling with the ablation guidewire.

\section{IMPACT ON LVOT OBSTRUCTION}

Figure 5 demonstrates LVOT obstruction after benchtop TMVR with and without preparatory LAMPOON. Figure 5F shows a typical post mortem result after in vivo LAMPOON. The 2 halves of the A2 leaflet are parted by the intact subvalvular apparatus. After benchtop TMVR, the LVOT anteroposterior diameters fell from $17 \pm 3$ to $5 \pm 4 \mathrm{~mm}$ (p $<0.01$ ) without LAMPOON and to $11 \pm 2 \mathrm{~mm}$ ( $\mathrm{p}<0.01)$ with LAMPOON. This represented a $69 \pm 18 \%$ reduction in LVOT diameter following TMVR compared with a 35 $\pm 10 \%$ reduction following LAMPOON $(\mathrm{p}<0.01)$. 


\section{DISCUSSION}

We demonstrate a new application for transcatheter electrosurgery to mitigate a lifethreatening complication of TMVR. Using simple catheter techniques, we can split the anterior mitral valve leaflet, which otherwise would be displaced anteriorly by the mitral valve implant and cause LVOT obstruction. The split leaflet edges are displaced around the transcatheter valve by chordal structures.

The potential for LVOT obstruction is a key barrier to TMVR and remains a devastating complication of early and investigational TMVR for native and post-annuloplasty mitral valve failure $(1,11,12)$. Imaging may predict risk for LVOT obstruction $(5,6,9)$ but their sensitivity and specificity remain uncertain. Nevertheless, it is clear that many patients are excluded from clinical and investigational transcatheter mitral valve therapy out of concern for iatrogenic LVOT obstruction.

LAMPOON may be especially helpful applied pro-phylactically in patients deemed at high risk for LVOT obstruction: those with unfavorable left ventricular geometry, acute aortomitral plane angulation, long leaflets, and a prominent septal bulge $(5,6,9)$. Without LAMPOON, operators may feel compelled to implant TMVR devices higher into the left atrium, which risks embolization. LAMPOON may allow lower implant position and more aggressive flaring of the implant, measures that otherwise would increase the risk for LVOT obstruction.

By comparison, intentional alcohol infarction to reduce interventricular septal thickness would best be performed in a separate procedure, is not suitable for patients with thin interventricular septa, and risks conduction injury and exacerbation of myocardial dysfunction (13).

The procedure sequence in patients would first be LAMPOON traversal and guidewire externalization, followed by pre-positioning of the TMVR device into the left atrium or unexpanded across the mitral valve, followed by LAMPOON laceration, followed by TMVR. Patients without baseline severe mitral valve regurgitation might be expected to experience severe hypotension between the laceration and implantation steps, which with proper planning could be achieved quickly.

\section{STUDY LIMITATIONS}

We use radiofrequency ablation to traverse and then lacerate the anterior mitral leaflet. Our traversal technique is the same as used to obtain transcaval access to the aorta $(14,15)$ and relies on radiofrequency power concentration on the guidewire monopole tip, further insulated by the Piggyback polymer jacket. For laceration, we overcome the tendency of charge to concentrate on the outer curvature of the intentionally kinked guidewire shaft by selectively denuding the inner curvature. During laceration, we minimize the length of exposed denuded guidewire by closely approximating the 2 guiding catheters. Both in vitro and in vivo, we observed no evidence of electric coupling to nearby conductive structures, which might heat the transcatheter mitral valve frame or hinder electrosurgical laceration (16). 
Many of the risks and limitations of LAMPOON are related to incorrect catheter placement and to electrosurgery. An eccentric or low crossing of the anterior leaflet would result in suboptimal parting of the leaflets. A high crossing may exit into the transverse sinus, risking pericardial effusion, but the guidewire can probably be withdrawn and the traversal repeated in the desired position. Entanglement with chordae could cause chordal injury or deleterious leaflet draping over the prosthetic valve and must be avoided by proper left atrial catheter positioning. We predict that 3-dimensional transesophageal echocardiography, of limited use in swine because of unfavorable anatomy, will be superior to 2-dimensional intracardiac echocardiography in guiding catheter position in patients. The risk for thrombus and gas embolism is mitigated by lower ablation energy and anticoagulation (17). Thrombus may form on the free edges of the lacerated leaflet, although we did not observe it, and may require post-procedural anticoagulation. There is a risk for bystander injury to the aortic valve, which is mitigated by proper insulation of the electrified guidewire. The role of LAMPOON is unclear for valve-in-valve TMVR, where chordal attachments are absent. Bailout LAMPOON is not an option in its current form, as the stent struts of the implanted valve prosthesis would prevent leaflet laceration.

This pre-clinical experience is limited to healthy juvenile swine, which unlike human patients have pristine noncalcified mitral leaflets and nontortuous aligned aortas and aortomitral structures. These structures may be more difficult to align and to lacerate in patients. Post mortem distortion of cardiac geometry probably confounds our ex vivo measurements of LVOT obstruction with and without LAMPOON. LAMPOON was not combined with TMVR in vivo in these animals, absent suitable valve devices. LAMPOON would appear better suited in combination with specific TMVR devices that do not rely on the intact anterior mitral leaflet for fixation. LAMPOON may induce hemodynamic compromise of different severity in human patients with pre-existing mitral valve disease and abnormal left atrial compliance.

\section{CONCLUSIONS}

LAMPOON has a promising role in therapy for patients ineligible for surgery and who have a risk for developing LVOT obstruction with TMVR. Serious risks can be mitigated by intraoperative echocardiographic guidance, adequate anticoagulation, and safe electrosurgical practice. Cautious application may be warranted in patients requiring TMVR expected to cause LVOT obstruction but who have no alternative options.

\section{Supplementary Material}

Refer to Web version on PubMed Central for supplementary material.

\section{Acknowledgments}

This work was supported by the Division of Intramural Research (Z01-HL006040), National Heart, Lung, and Blood Institute, National Institutes of Health. Dr. Greenbaum serves as a proctor for Edwards Lifesciences and St. Jude Medical, which manufactures transcatheter heart valves. Dr. Babaliaros is a consultant for Edwards Lifesciences and Abbott Vascular; and his employer has research contracts for multicenter investigation of transcatheter aortic and mitral devices from Edwards Lifesciences, Abbott Vascular, Medtronic, St. Jude Medical, and Boston Scientific. 
The authors thank Alan Hoofring of the National Institutes of Health Division of Medical Arts and Katherine Lucas, Shawn Koslov, and Joni Taylor from the National Heart, Lung, and Blood Institute's Animal Surgery and Resources Core.

\section{ABBREVIATIONS AND ACRONYMS}

LAMPOON laceration of the anterior mitral leaflet to prevent left ventricular outflow tract obstruction

LVOT left ventricular outflow tract

TMVR transcatheter mitral valve implantation

\section{References}

1. Eleid MF, Cabalka AK, Williams MR, et al. Percutaneous transvenous transseptal trans-catheter valve implantation in failed bioprosthetic mitral valves, ring annuloplasty, and severe mitral annular calcification. J Am Coll Cardiol Intv. 2016; 9:1161-74.

2. Sud K, Agarwal S, Parashar A, et al. Degenerative mitral stenosis: unmet need for percutaneous interventions. Circulation. 2016; 133:1594-604. [PubMed: 27142604]

3. Lee KS, Stewart WJ, Lever HM, Underwood PL, Cosgrove DM. Mechanism of outflow tract obstruction causing failed mitral valve repair. Anterior displacement of leaflet coaptation. Circulation. 1993; 88:II24-9. [PubMed: 8222161]

4. Mihaileanu S, Marino JP, Chauvaud S, et al. Left ventricular outflow obstruction after mitral valve repair (Carpentier's technique). Proposed mechanisms of disease. Circulation. 1988; 78:I78-84. [PubMed: 3409521]

5. Blanke P, Naoum C, Dvir D, et al. Predicting LVOT obstruction in transcatheter mitral valve implantation: concept of the neo-LVOT. J Am Coll Cardiol Img. In press.

6. Wang DD, Eng M, Greenbaum A, et al. Predicting LVOT obstruction after TMVR. J Am Coll Cardiol Img. In press.

7. Maisano F, Alfieri $\mathrm{O}$, Banai $\mathrm{S}$, et al. The future of transcatheter mitral valve interventions: competitive or complementary role of repair vs. replacement? Eur Heart J. 2015; 36:1651-9. [PubMed: 25870204]

8. Deharo P, Urena M, Himbert D, et al. Bail-out alcohol septal ablation for left ventricular outflow tract obstruction after transcatheter mitral valve replacement. J Am Coll Cardiol Intv. 2016; 9:e736.

9. Guerrero M, Urena M, Pursnani A, et al. Balloon expandable transcatheter heart valves for native mitral valve disease with severe mitral annular calcification. J Cardiovasc Surg. 2016; 57:401-9. [PubMed: 27094423]

10. Guerrero M, Dvir D, Himbert D, et al. Trans-catheter mitral valve replacement in native mitral valve disease with severe mitral annular calcification: results from the first multicenter global registry. J Am Coll Cardiol Intv. 2016; 9:1361-71.

11. Descoutures F, Himbert D, Maisano F, et al. Transcatheter valve-in-ring implantation after failure of surgical mitral repair. Eur J Cardiothorac Surg. 2013; 44:e8-15. [PubMed: 23530029]

12. Bouleti C, Fassa AA, Himbert D, et al. Transfemoral implantation of transcatheter heart valves after deterioration of mitral bioprosthesis or previous ring annuloplasty. J Am Coll Cardiol Intv. 2015; 8:83-91.

13. Gersh BJ, Maron BJ, Bonow RO, et al. 2011 ACCF/AHA guideline for the diagnosis and treatment of hypertrophic cardiomyopathy. J Am Coll Cardiol. 2011; 58:2703-38. [PubMed: 22075468]

14. Halabi M, Ratnayaka K, Faranesh AZ, Chen MY, Schenke WH, Lederman RJ. Aortic access from the vena cava for large caliber transcatheter cardiovascular interventions: pre-clinical validation. $J$ Am Coll Cardiol. 2013; 61:1745-6. [PubMed: 23500317] 
15. Greenbaum AB, O'Neill WW, Paone G, et al. Caval-aortic access to allow transcatheter aortic valve replacement in otherwise ineligible patients: initial human experience. J Am Coll Cardiol. 2014; 63:2795-804. [PubMed: 24814495]

16. Robinson TN, Barnes KS, Govekar HR, Stiegmann GV, Dunn CL, McGreevy FT. Antenna coupling - a novel mechanism of radiofrequency electrosurgery complication: practical implications. Ann Surg. 2012; 256:213-8. [PubMed: 22791097]

17. Takami M, Lehmann HI, Parker KD, Welker KM, Johnson SB, Packer DL. Effect of left atrial ablation process and strategy on microemboli formation during irrigated radiofrequency catheter ablation in an in vivo model. Circ Arrhythm Electrophysiol. 2016; 9:e003226. [PubMed: 26763224] 


\section{PERSPECTIVES}

\section{WHAT IS KNOWN?}

TMVR risks life-threatening LVOT obstruction by displacing the anterior mitral leaflet.

\section{WHAT IS NEW?}

LAMPOON is a catheter technique to transect the anterior mitral leaflet, to prevent iatrogenic LVOT obstruction.

\section{WHAT IS NEXT?}

On the basis of this series of pre-clinical experiments, LAMPOON may be ready for cautious investigation in selected patients at high risk for LVOT obstruction. 


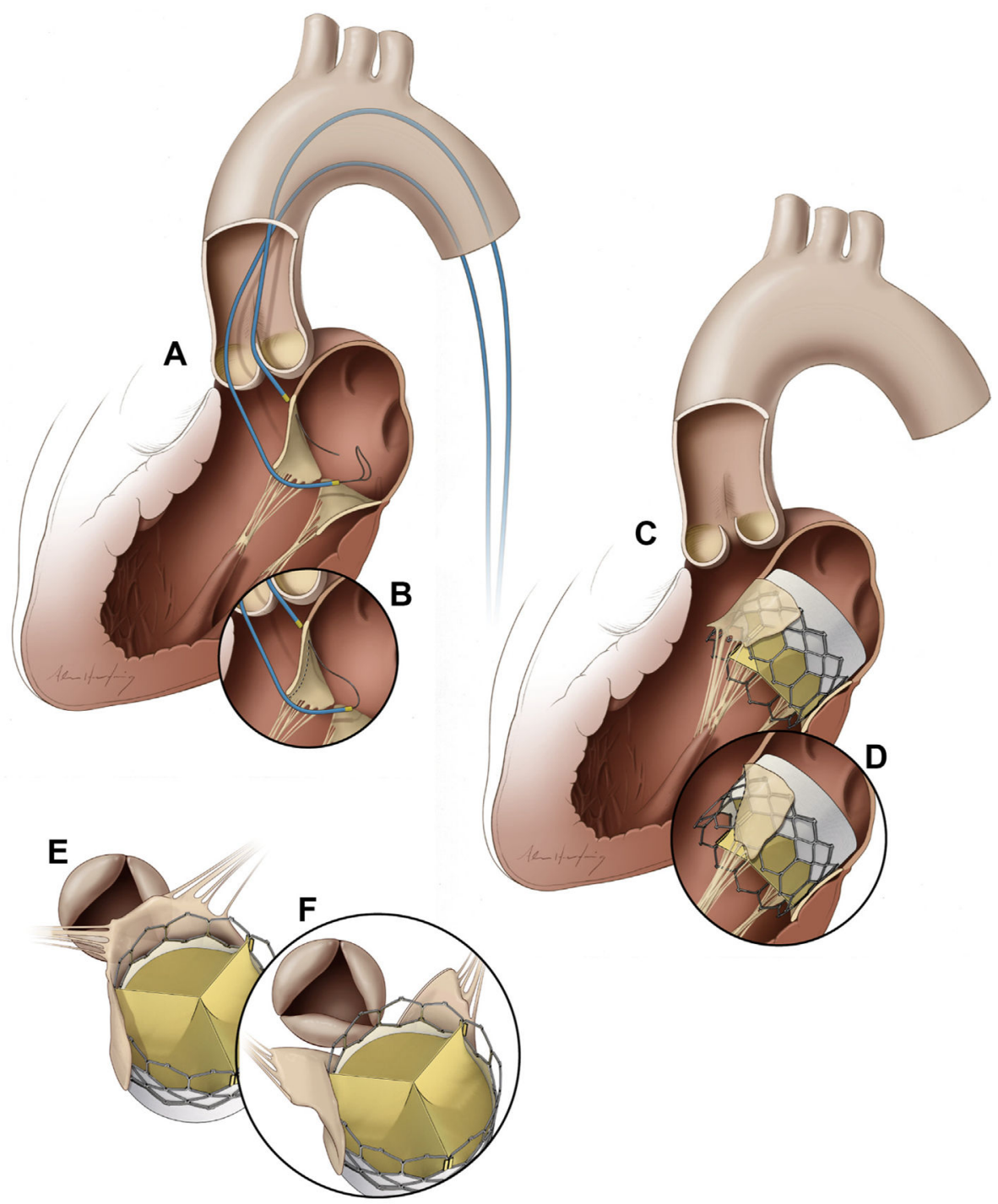

FIGURE 1. Illustrations of the Technique of Intentional Laceration of the Anterior Mitral Valve Leaflet to Prevent Left Ventricular Outflow Tract Obstruction

(A) Two Judkins left catheters are positioned on either side of the A2 mitral leaflet base. An energized guidewire is advanced from the left ventricular outflow tract (LVOT) catheter into the left atrial catheter snare. (B) The snared tip is externalized to form a guidewire loop around the A2 leaflet. This is energized and pulled outward to lacerate the leaflet lengthwise into 2 halves. (C,E) A transcatheter mitral valve implant tents the anterior mitral leaflet into the septum, obstructing the LVOT. (D,F) Splitting the leaflet by intentional laceration of the anterior mitral valve leaflet to prevent LVOT obstruction instead causes the 2 tethered halves to displace along either side of the transcatheter valve, preventing LVOT obstruction. 


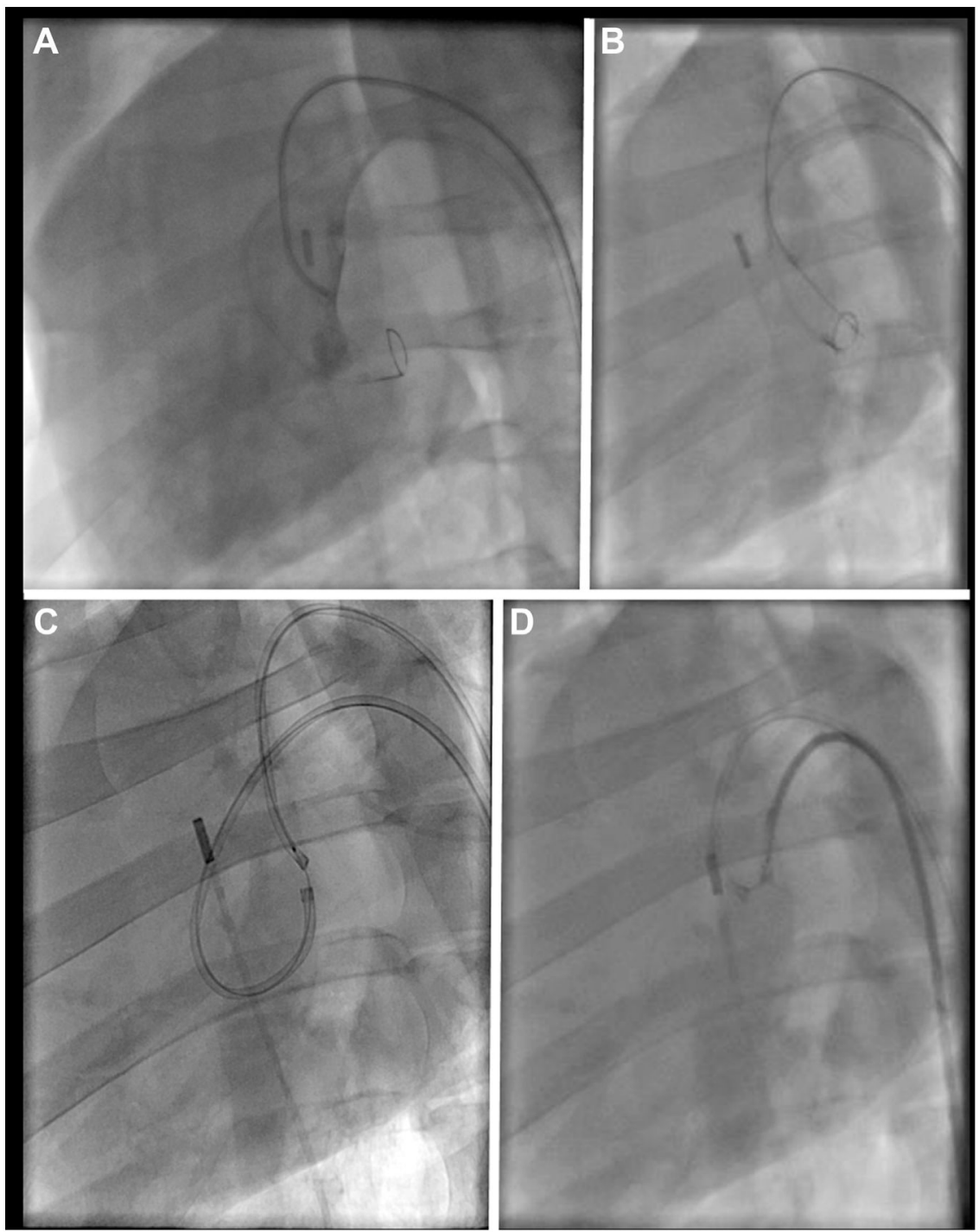

FIGURE 2. Fluoroscopy Demonstration of Intentional Laceration of the Anterior Mitral Valve Leaflet to Prevent Left Ventricular Outflow Tract Obstruction in a Left Oblique Projection (A) Angiography through the left ventricular outflow tract (LVOT) catheter shows good positioning of this catheter at the base of the anterior leaflet, below the aortic valve, with a loop snare positioned through the left atrial (LA) catheter. (B) The electrified guidewire is advanced through the A2 mitral leaflet base into the LA snare. (C) A denuded kinked section of the guidewire, insulated and marked proximally with a polymer wire convertor further insulated by the 2 guiding catheters, is electrified while the LA catheter is pulled back into the LVOT (position D) during stage 1 of the 2-step electrosurgical laceration. (D) Stage 2 of the laceration. Both catheters are pulled in tandem during a burst application of radiofrequency energy, lacerating the leaflet completely and freeing the catheter-guidewire loop. 


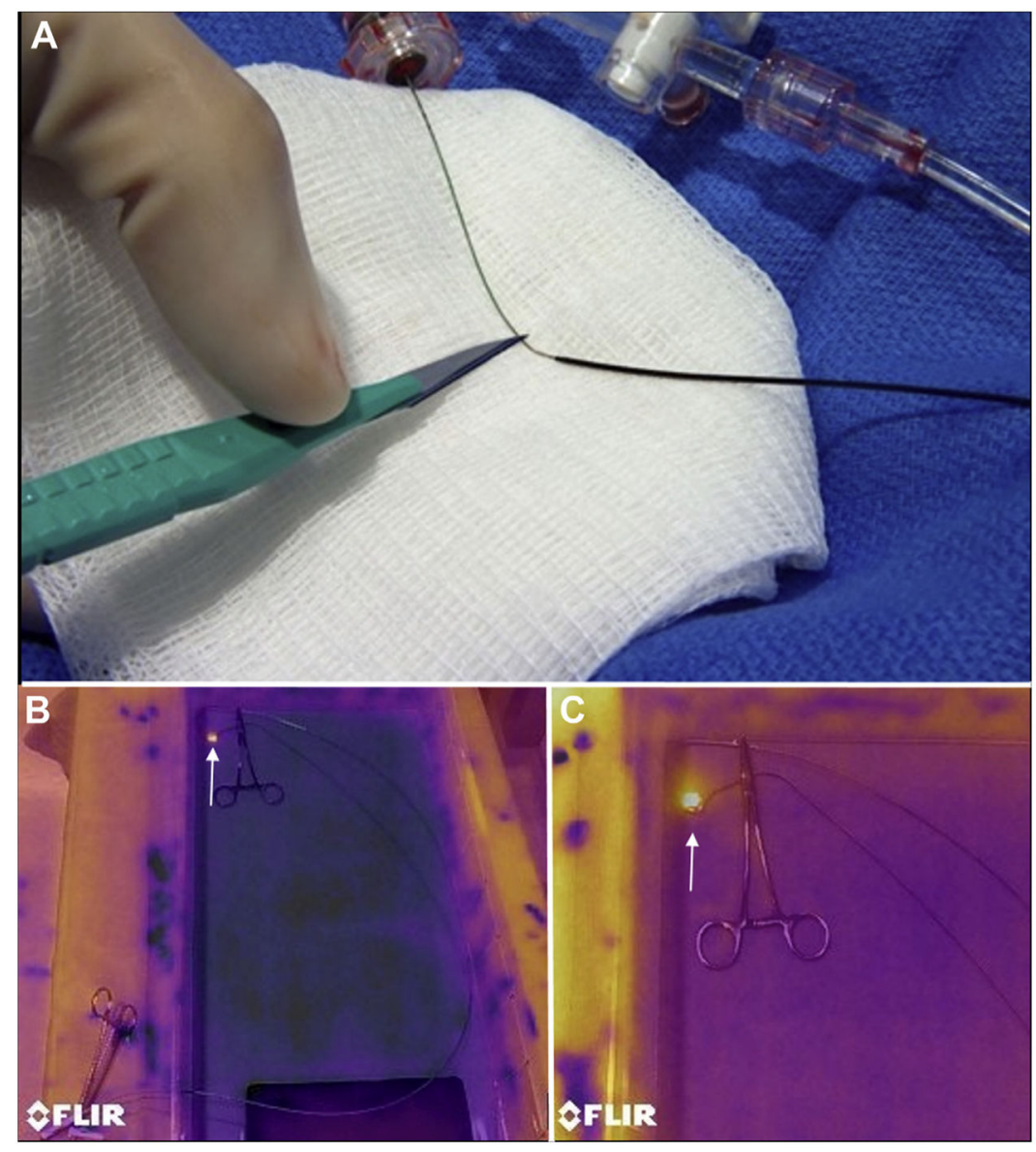

FIGURE 3. Guidewire Electrosurgery

(A) A short midshaft section of the electrically insulating polytetrafluoroethylene coating of a 0.014-inch guidewire is stripped using a scalpel and then kinked, with a polymer jacket wire convertor locked alongside. (B) Infrared images of a saline bath with a denuded guidewire loop through 2 catheters, replicating in vivo intentional laceration of the anterior mitral valve leaflet to prevent left ventricular outflow tract obstruction. The guidewire is clipped to an electrosurgery pencil and electrified, revealing a hot spot (bright yellow, arrow) only at the exposed guidewire loop. (C) A close-up of the guidewire loop reveals no heating around the nearby metallic hemostat, suggesting freedom from electric coupling. 


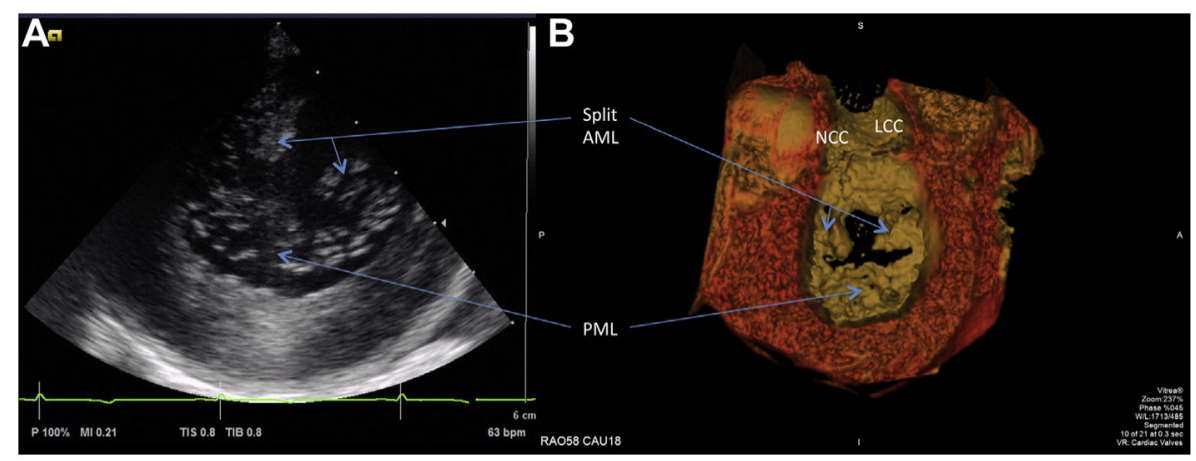

FIGURE 4. Images of the Lacerated Anterior Mitral Leaflet After Intentional Laceration of the Anterior Mitral Valve Leaflet to Prevent Left Ventricular Outflow Tract Obstruction

(A) Short-axis intraoperative intracardiac echocardiographic image of the mitral valve showing the anterior mitral leaflet (AML) split in 2 equal halves. (B) The corresponding post-procedural surface rendering of contrast-enhanced computed tomography also displaying split and splayed leaflets. See Online Video 1. AML = anterior mitral leaflet; LCC = left coronary cusp; NCC = noncoronary cusp; PML = posterior mitral leaflet. 


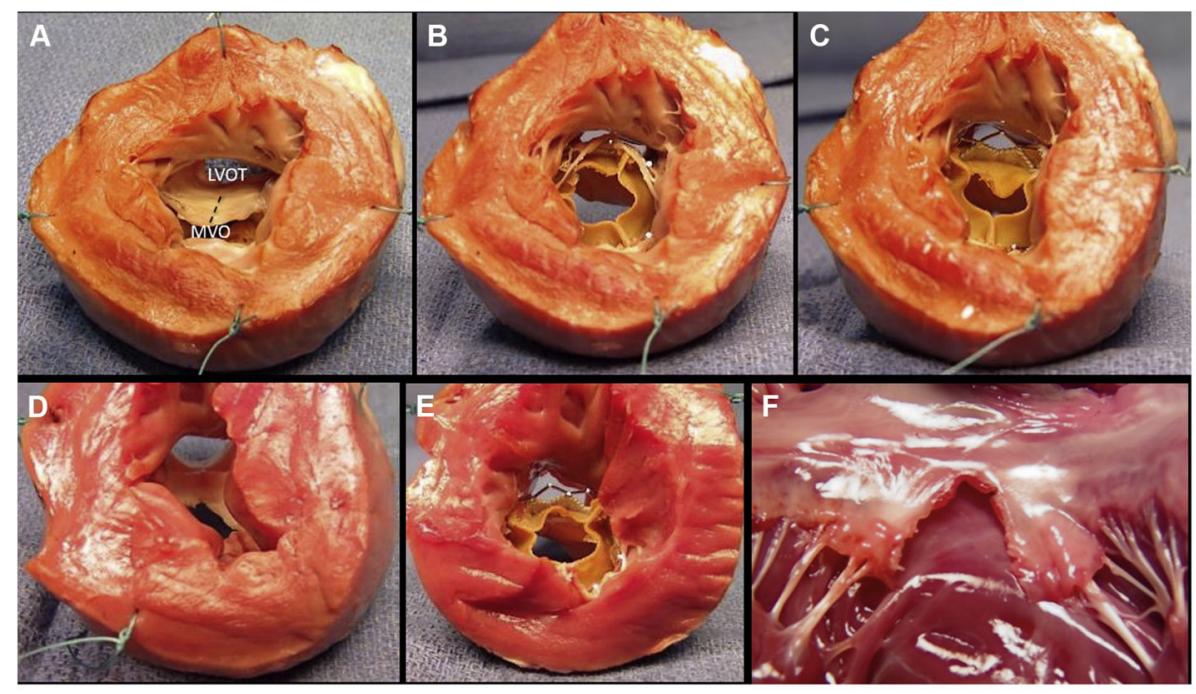

FIGURE 5. Benchtop Assessment of Left Ventricular Outflow Tract Geometry Impact

The base of the left ventricle is viewed in cross section after the apex is cut away. (A) A naive heart with the anterior mitral leaflet intact. The trajectory of an intentional laceration of the anterior mitral valve leaflet to prevent left ventricular outflow tract obstruction (LAMPOON) laceration is depicted by the dashed line. (B) Transcatheter mitral valve replacement (TMVR) with intact anterior leaflet showing reduced left ventricular outflow tract (LVOT) area. (C) LAMPOON modification made to the same heart, with the anterior leaflets displaced to the side by TMVR and reduced LVOT obstruction. Flow would be possible through uncovered stent struts. (D) Explant after in vivo LAMPOON heart showing lacerated anterior leaflet. (E) TMVR in the explanted heart after LAMPOON showing displacement of the anterior leaflet away from the LVOT. (F) Explanted heart after isolated in vivo LAMPOON viewed from the posterior wall, showing central laceration down the complete length of the anterior leaflet. The intact subvalvular apparatus displaces the leaflet tips away from the LVOT. LVOT = left ventricular outflow tract; MVO = mitral valve orifice. 